\title{
Evaluation of Four Lymph Node Classifications for the Prediction of Survival in Hilar Cholangiocarcinoma
}

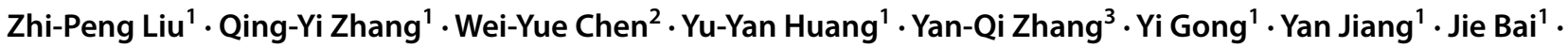 \\ Zhi-Yu Chen ${ }^{1} \cdot$ Hai-Su Dai ${ }^{1}$ (]
}

Received: 16 September 2021 / Accepted: 20 November 2021 / Published online: 1 January 2022

(c) The Author(s) 2021

\begin{abstract}
Background An important prognostic indicator of hilar cholangiocarcinoma (HCCA) in patients after surgery is metastasis of lymph nodes (LN). However, there are many types of LN staging systems to the issue of a better determination of the prognosis of patients through the lymphatic staging system which needs research. Based on the above, we tried to re-evaluate the staging system of HCCA LNs. We compared the American Joint Committee on Cancer (AJCC), number of metastatic LNs (MLN), ratio of LN (LNR), and log odds of MLNs (LODDS) in individuals undergoing curative resection to determine the best LN staging system.

Methods In the current study, we retrospectively analyzed 229 patients undergoing curative resection. We evaluated the impact of the stage of AJCC pN, LNR, LODDS, and MLN on OS (overall survival) and RFS (recurrence-free survival). According to the curve of receiver operating characteristic (ROC), we compared the predictive capacity of different staging systems of LN for survival and recurrence.

Results Multivariate analysis results revealed that LODDS $>-0.45(95 \% \mathrm{CI}=1.115-2.709, P=0.015 ; 95 \% \mathrm{CI}=1.187-$ 2.780, $P=0.006$ ) are independent risk factors affecting OS and RFS, respectively. Compared with LN status, AJCC pN stage, MLN, and LNR, the variable having the highest area under the ROC curve (AUC) was LODDS when predicting 1-year, 3-year, and 5-year OS and RFS.

Conclusion This study shows that metastasis of LNs is a key indicator for predicting patient death and recurrence. Among them, LODDS is the best LN staging system for the prognostic evaluation of HCCA patients after surgery. Clinicians can incorporate LODDS into HCCA patient lymphatic staging system for a more accurate prognosis of HCCA patients post-surgery.
\end{abstract}

Keywords Hilar cholangiocarcinoma $\cdot$ Lymph node metastasis $\cdot$ Lymph node staging system

Zhi-Peng Liu and Qing-Yi Zhang contributed equal to this work.

Zhi-Yu Chen

chenzhiyu_umn@163.com

$\checkmark$ Hai-Su Dai

daihaisu@163.com

1 Department of Hepatobiliary Surgery, Southwest Hospital, Third Military Medical University (Army Medical University), No. 30 Gaotanyan Road, Chongqing 400038, China

2 Department of Clinical Center of Oncology, Zhejiang University Lishui Hospital, Lishui 323000, Zhejiang, China

3 Department of Health Statistics, College of Military Preventive Medicine, Army Medical University, Chongqing, China

\section{Introduction}

Hilar cholangiocarcinoma (HCCA) is a highly malignant tumor with an increasing incidence in recent years ${ }^{1}$. The prognosis of HCCA is usually poor, so an accurate prognosis is extremely important. Metastasis to lymph nodes $(\mathrm{LN})$ is a key tumor prognosis indicator ${ }^{2,3}$. The prognosis of most tumors is directly related to metastasis of the $\mathrm{LN}$ and their number. Studies have shown that more than $30 \%$ of patients with HCCA who underwent curative resection have LN metastasis ${ }^{4-6}$. However, it is still unknown which $\mathrm{LN}$ staging system is in use to judge patient prognosis more accurately.

To better describe the status of LNs, some scholars use different methods to classify LN metastases ${ }^{7,8}$. Among 
them, the pN stage as per the American Joint Committee on Cancer (AJCC) staging is based on incremental tumorinfiltrated positive $\mathrm{LNs}^{9,10}$. $\mathrm{LN}$ ratio (LNR) is defined as the number of positive lymph nodes divided by the total number of lymph nodes examined ${ }^{8}$. Studies have shown that the LNR is superior to the number of metastatic LNs (MLN) for staging in cholangiocarcinoma ${ }^{11-13}$. However, LN staging systems such as LNR, MLN, and AJCC pN stage have limitations in predicting the prognosis of patients 14. In recent years, the log odds of MLNs (LODDS) has been included by clinicians as a new prognostic indicator ${ }^{15}$. LODDS were calculated as the log of $[M L N \pm 0.5 /$ total number of LNs retrieved (TLN) $-\mathrm{MLN}+0.5]{ }^{16}$. Kwon et al. have confirmed that LODDS is the best LN staging system for judging the prognosis of patients with ampullary adenocarcinoma ${ }^{17}$. Zhou et al. used MLN, LNR, LODDS, and other LN staging systems to assess the prognosis of patients with distant metastatic cancer of the stomach and found that LODDS is the most accurate system of LN staging for assessing the prognosis of patients ${ }^{18}$. Conci et al. evaluated the LN analysis system of 99 HCCA patients and pointed out that LODDS is the best LN staging system for the prediction of the overall survival (OS) time of patients ${ }^{19}$. However, his study did not compare the performance of the LN staging system in assessing HCCA recurrence.

Thus, this study aimed to compare MLN, LNR, AJCC $\mathrm{pN}$ stage values, and LODDS in evaluating the OS and recurrence-free survival (RFS) of patients with HCCA who underwent curative resection and finally determine the most suitable LN staging system.

\section{Methods}

\section{Patient Selection}

We retrospectively evaluated the medical records of 229 newly diagnosed HCCA patients who underwent curative resection in Southwest Hospital of China from January 2006 to December 2019. Each patient was confirmed to be HCCA by postoperative pathology. Patients who were found to be unresectable at exploration, had received adjuvant chemotherapy and radiotherapy, and died within 30 days after surgery were excluded. Curative resection was defined as complete resection of all macroscopic and microscopic HCCA tumor with microscopically clear resection margins in the surgical specimens. Regardless of preoperative computed tomography (CT) or magnetic resonance imaging (MRI) or suspicion of lymph node metastasis, patients underwent loco-regional lymphadenectomy. All lymph node tissue underwent pathological biopsy. This research followed the ethical guidelines of the World Medical Association (WMA; Declaration of Helsinki). The approval for the present research was obtained from the Ethics Committee of Southwest Hospital (approval number KY2021129), and all patients obtained informed, written consent for the clinical study.

\section{Outcome Measures}

The distribution of tumors along the bile duct during our operation is classified as per Bismuth-Corlette ${ }^{20}$. Classification of the LN location is based on the English version of the 3rd edition of the Japanese Cholangiocarcinoma Classification ${ }^{21}$. As per the AJCC staging system (8th edition), the LN metastasis involved in the common bile duct, cystic duct, hepatic artery, and portal vein is $\mathrm{N} 1$; the metastatic $\mathrm{LN}$ involved in the mesentery, abdominal cavity, and para-abdominal aorta is $\mathrm{N} 2{ }^{9}$. LNR is the ratio of the MLN/TLN ${ }^{11}$. Based on the outcomes of earlier studies, patients were divided into intervals of LNR as LNR0, LNR =0; LNR1, $0.01<\mathrm{LNR} \leq 0.25$; and LNR2, LNR $>0.25^{11}$. The influence of the number of MLN on the prognosis is ascertained by values for cut-off as $0,1 \sim 3$, and $>3{ }^{19}$. LODDS is defined as $\log [(\mathrm{MLN}+0.5)$ divided by $(\mathrm{TLN}-\mathrm{MLN}+0.5)]^{22}$. We defined the intervals of the LODDS LN staging system for HCCA patients according to previous studies but found that the proportion of patients in each interval of LODDS varied remarkably ${ }^{19}$. Therefore, we performed three equal categorizations according to the numerical order of LODDS. That is, all patients were categorized into the following three LODDS intervals: LODDS 1, LODDS $\leq-0.85$; LODDS2, LODDS $-0.85<$ LODDS $\leq-0.45$; and LODDS3, LODDS $>-0.45$.

The focus of this study was OS and RFS. OS was defined from the surgery date to the last follow-up or death due to any cause. The definition of RFS was the time from the surgery date to the diagnosis of recurrence or no disease found at the last follow-up. If the patient did not relapse, the definition of RFS was from the surgery date to the last follow-up or the date of death.

\section{Statistical Analysis}

For continuous variables, median and IQR (interquartile range) or the mean and standard deviation were used. Fisher's exact test or the chi-square test was applied for categorical variables, and Mann-Whitney $U$ test was implemented for numerical variables. We used $25 \mathrm{~mm}$ and $150 \mathrm{U} / \mathrm{L}$ as the cut-off value of tumor size and CA19-9 ${ }^{19,23}$. The univariate survival rate was determined using the Kaplan-Meier method, and for comparison, the log-rank test was applied. We used the model of Cox regression for multivariate assessments to ascertain independent prognostic criteria. We analyzed the factors having $P<0.10$ in the univariate analysis as covariates in multivariate analysis. In addition, we calculated risk ratios (HRs) and 95\% confidence intervals 
(CIs), and $\mathrm{HR}<1.0$ indicates a survival benefit. For an accurate evaluation and comparison of the prognostic ability of different methods of LN staging, we used receiver operating characteristics curve (ROC) and area under the ROC curve (AUC) to compare LODDS, LNR, MLN, AJCC pN stage, LN status, and other indicators in predicting the difference in the mortality and recurrence rate of patients at each time point. Statistical analyses were performed using SPSS ${ }^{\circledR}$ version 26.0 (IBM, Armonk, New York, USA).

\section{Results}

\section{Basic Clinical Information}

The demographic, clinical, and pathological data of 246 HCCA patients who had surgery in the period between
January 2006 and December 2019 were collected. Among these patients, 229 patients had complete pathological and clinical data, so they were included in the current survey. A summary of study population data was summarized in Table 1. One hundred fifty-two cases $(66.4 \%)$ underwent major live resection. All patients underwent loco-regional lymphadenectomy. There were 143 cases $(62.4 \%)$ in AJCC pN0, 63 cases $(27.5 \%)$ in AJCC pN1, and 23 cases (10.1\%) in AJCC pN2. The median number of LNs examined is 4 (IQR, 1-20); among them, 93 patients (40.6\%) took 1-3, 126 patients $(55.0 \%)$ took $4-10$, and 10 patients $(4.4 \%)$ took more than 10. A total of 86 patients (37.6\%) had MLN. The median of MLN was 2 (IQR, 1-9). The MLN of 71 cases $(31.0 \%)$ was $1-3$, and the MLN of 16 cases $(6.6 \%)$ was $>3$. The median of LNR and LODDS were 0.00 (IQR, $0.00-1.00)$ and -0.70 (IQR, $-1.52-1.18$ ), respectively.
Table 1 Clinical and pathological characteristics of 229 patients with hilar cholangiocarcinoma resected with curative intent

\begin{tabular}{|c|c|c|}
\hline \multicolumn{2}{|l|}{ Characteristics } & Values \\
\hline \multicolumn{2}{|l|}{ Age, years, median (IQR) } & $58(49-66)$ \\
\hline \multicolumn{2}{|l|}{ Size, mm, median (IQR) } & $3.0(2.0-3.8)$ \\
\hline \multicolumn{2}{|c|}{ Preoperative CA19-9, U/L, median (IQR) } & $186.1(60.0-459.0)$ \\
\hline \multirow[t]{2}{*}{ Gender, $n(\%)$} & Male & $137(59.8 \%)$ \\
\hline & Female & $92(40.2 \%)$ \\
\hline \multirow[t]{2}{*}{ Type of hepatectomy, $n(\%)$} & Minor & $77(33.6 \%)$ \\
\hline & Major & $152(66.4 \%)$ \\
\hline \multirow[t]{2}{*}{ Vascular invasion, $n(\%)$} & No & $136(59.4 \%)$ \\
\hline & Yes & $93(40.6 \%)$ \\
\hline \multirow[t]{2}{*}{ Perineural invasion, $n(\%)$} & No & $147(64.2 \%)$ \\
\hline & Yes & $82(35.8 \%)$ \\
\hline \multirow[t]{2}{*}{ Differentiation, $n(\%)$} & Well-moderated & $40(17.5 \%)$ \\
\hline & Poor & $189(82.5 \%)$ \\
\hline \multirow[t]{2}{*}{ Bismuth type, $n(\%)$} & $\mathrm{I}-\mathrm{II}$ & $98(42.8 \%)$ \\
\hline & III-IV & $131(57.2 \%)$ \\
\hline \multirow[t]{3}{*}{ AJCC pN stage, $n(\%)$} & 0 & $143(62.4 \%)$ \\
\hline & 1 & $63(27.5 \%)$ \\
\hline & 2 & $23(10.1 \%)$ \\
\hline \multirow[t]{3}{*}{ Number LN retrieved, $n(\%)$} & $1-3$ & $93(40.6 \%)$ \\
\hline & $4-10$ & $126(55.0 \%)$ \\
\hline & $>10$ & $10(4.4 \%)$ \\
\hline \multirow[t]{3}{*}{ MLN, $n(\%)$} & 0 & $143(62.4 \%)$ \\
\hline & $1-3$ & $71(31.0 \%)$ \\
\hline & $>3$ & $15(6.6 \%)$ \\
\hline \multirow[t]{3}{*}{$\mathrm{LNR}, n(\%)$} & LNR0 (0) & $143(62.4 \%)$ \\
\hline & LNR1 (0.01-0.25) & $29(12.7 \%)$ \\
\hline & LNR2 (>0.25) & $57(24.9 \%)$ \\
\hline \multirow[t]{3}{*}{ LODDS, $n(\%)$} & LODDS1 $(\leq-0.85)$ & $75(32.8 \%)$ \\
\hline & LODDS2 $(-0.84--0.45)$ & $90(39.3 \%)$ \\
\hline & LODDS3 $(>-0.45)$ & $64(27.9 \%)$ \\
\hline
\end{tabular}

$I Q R$, interquartile range; $L N$, lymph node; CA19-9, carbohydrate antigen 19-9; AJCC, American Joint Committee on Cancer; $M L N$, number of metastatic LNs; $L N R$, lymph node ratio; $L O D D S$, log odds of metastatic lymph node 


\section{Survival Analysis}

The OS for 1,3 , and 5 years of the total study population were $73.5 \%, 32.4 \%$, and $23.0 \%$, respectively, and the median OS time was 23.0 months ( $95 \% \mathrm{CI}=19.4-26.6$ months). The RFS of 1,3 , and 5 years of the total study population were $54.6 \%, 25.1 \%$, and $16.8 \%$, respectively, and the median RFS was 16.0 months $(95 \% \mathrm{CI}=13.0-19.0$ months).

\section{Analysis of Risk factors Affecting Survival}

The study analyzed the clinical and pathological factors that affected OS. The level of CA19-9 ( $<500 \mathrm{vs} \geq 500 \mathrm{U} / \mathrm{L}$, $P=0.083)$, size of tumors $(<2.5 \mathrm{vs} \geq 2.5 \mathrm{~cm}, P=0.002)$, vascular invasion (no vs yes, $P=0.009$ ), degree of differentiation (medium/well-differentiated compared to poorly differentiated, $P<0.001$ ), and LN metastasis (negative vs positive, $P=0.010$ ) are the primary risk factors that affect OS in univariate analysis (Table 2).

We analyzed the clinical and pathological factors affecting RFS. The level of CA19-9 ( $<500 \mathrm{vs} \geq 500 \mathrm{U} / \mathrm{L}$, $P=0.092)$, size of tumor $(<2.5 \mathrm{vs} \geq 2.5 \mathrm{~cm}, P=0.003)$, vascular invasion (no vs. yes, $P=0.037$ ), degree of differentiation (medium/highly differentiated compared to poorly differentiated, $P \leq 0.003$ ), and $\mathrm{LN}$ metastasis (negative vs positive, $P=0.001$ ) were the primary risk factors affecting RFS in univariate analysis (Table 3 ).

AJCC pN stage (OS, $P=0.012$, Fig. 1A; RFS, $P=0.001$, Fig. 1B), MLN (OS, $P=0.034$, Fig. $1 \mathrm{C}$; RFS, $P=0.002$, Fig. 1D), LNR (OS, $P=0.024$, Fig. 1E; RFS, $P=0.005$, Fig. 1F), LODDS (OS, $P=0.002$, Fig. $1 \mathrm{G}$; RFS, $P<0.001$, Fig. $1 \mathrm{H}$ ), and other LN staging systems to group HCCA patients. The results indicate that OS and RFS differed significantly in HCCA patients.

The CA19-9 level, vascular invasion, method of resection, tumor size, and degree of differentiation were included as independent variables into the multivariate Cox analysis, and different LN staging methods were used to perform multivariate survival analysis on OS and RFS. Among them, positive $\mathrm{LN}$ status $(95 \% \mathrm{CI}=1.125-1.917$, $P=0.041)$, AJCC pN2 (95\% CI $=1.001-2.940, P=0.049)$, LNR $>0.25(95 \% \mathrm{CI}=1.018-2.250, P=0.040)$, and LODDS $>-0.45(95 \% \mathrm{CI}=1.115-2.709, P=0.015)$ were independent risk factors affecting OS (Table 4). LN status positive $(95 \% \mathrm{CI}=1.073-2.059, P=0.017)$, AJCC pN2 (95\% CI $=1.138-3.143, P=0.014)$, MLN $1-3$ $(95 \% \mathrm{CI}=1.052-2.078, P=0.024), \mathrm{LNR}>0.25(95 \%$ $\mathrm{CI}=1.030-2.200, P=0.035)$, and LODDS $>-0.45(95 \%$
Table 2 Kaplan-Meier analysis of the association between overall survival and clinical and pathological factors in the 229 study patients

\begin{tabular}{|c|c|c|c|c|c|c|}
\hline \multirow[t]{2}{*}{ Prognostic factors } & & \multicolumn{5}{|c|}{ Kaplan-Meier univariate analysis } \\
\hline & & $\begin{array}{l}\text { Median OS } \\
\text { (months) }\end{array}$ & 1-year OS & 3-year OS & 5-year OS & $P$ \\
\hline \multirow[t]{2}{*}{ Age } & $<60$ & 23.0 & $76.8 \%$ & $37.4 \%$ & $25.7 \%$ & .221 \\
\hline & $\geq 60$ & 21.0 & $69.5 \%$ & $27.1 \%$ & $19.8 \%$ & \\
\hline \multirow[t]{2}{*}{ Gender } & Male & 24.0 & $74.2 \%$ & $33.7 \%$ & $22.9 \%$ & .700 \\
\hline & Female & 19.0 & $72.6 \%$ & $30.7 \%$ & $23.3 \%$ & \\
\hline \multirow[t]{2}{*}{ CA19-9 } & $<500 \mathrm{U} / \mathrm{L}$ & 31.0 & $78.7 \%$ & $42.2 \%$ & $24.4 \%$ & .083 \\
\hline & $\geq 500 \mathrm{U} / \mathrm{L}$ & 21.0 & $70.9 \%$ & $27.7 \%$ & $22.1 \%$ & \\
\hline \multirow[t]{2}{*}{ Tumor size } & $<25 \mathrm{~mm}$ & 26.0 & $78.3 \%$ & $39.8 \%$ & $31.1 \%$ & .002 \\
\hline & $\geq 25 \mathrm{~mm}$ & 17.0 & $68.5 \%$ & $24.7 \%$ & $14.2 \%$ & \\
\hline \multirow[t]{2}{*}{ Bismuth type } & $\mathrm{I}-\mathrm{II}$ & 22.0 & $74.6 \%$ & $36.5 \%$ & $25.3 \%$ & .372 \\
\hline & III-IV & 24.0 & $72.2 \%$ & $27.4 \%$ & $19.8 \%$ & \\
\hline \multirow[t]{2}{*}{ Type of hepatectomy } & Minor & 23.0 & $72.3 \%$ & $32.0 \%$ & $21.7 \%$ & .875 \\
\hline & Major & 22.0 & $74.2 \%$ & $32.6 \%$ & $23.6 \%$ & \\
\hline \multirow[t]{2}{*}{ Vascular invasion } & No & 25.0 & $78.4 \%$ & $38.6 \%$ & $25.6 \%$ & .009 \\
\hline & Yes & 16.0 & $66.5 \%$ & $22.2 \%$ & $19.4 \%$ & \\
\hline \multirow[t]{2}{*}{ Perineural invasion } & No & 24.0 & $74.0 \%$ & $34.9 \%$ & $24.3 \%$ & .227 \\
\hline & Yes & 18.0 & $72.8 \%$ & $27.9 \%$ & $19.9 \%$ & \\
\hline \multirow[t]{2}{*}{ Differentiation } & Well-moderated & 25.0 & $79.2 \%$ & $36.8 \%$ & $25.6 \%$ & $<.001$ \\
\hline & Poor & 12.0 & $46.6 \%$ & $10.8 \%$ & $10.8 \%$ & \\
\hline \multirow[t]{2}{*}{ LN status } & Negative (N0) & 25.0 & $77.4 \%$ & $38.1 \%$ & $28.5 \%$ & .010 \\
\hline & Positive $(\mathrm{N}+)$ & 18.0 & $67.1 \%$ & $22.6 \%$ & $12.6 \%$ & \\
\hline
\end{tabular}

$P, P$ value of the log-rank test

OS, overall survival; $C A 19-9$, carbohydrate antigen $19-9 ; L N$, lymph node 
Table 3 Kaplan-Meier analysis and multivariable Cox regression survival analysis of the association between recurrence-free survival and clinical and pathological factors in the 229 study patients

\begin{tabular}{|c|c|c|c|c|c|c|}
\hline \multirow[t]{2}{*}{ Prognostic factors } & & \multicolumn{5}{|c|}{ Kaplan-Meier univariate analysis } \\
\hline & & $\begin{array}{l}\text { Median RFS } \\
\text { (months) }\end{array}$ & 1-year RFS & 3-year RFS & 5-year RFS & $P$ \\
\hline \multirow[t]{2}{*}{ Age } & $<60$ & 14.0 & $52.7 \%$ & $27.8 \%$ & $24.8 \%$ & .653 \\
\hline & $\geq 60$ & 17.0 & $56.8 \%$ & $22.4 \%$ & $10.9 \%$ & \\
\hline \multirow[t]{2}{*}{ Gender } & Male & 17.0 & $57.2 \%$ & $25.1 \%$ & $17.0 \%$ & .590 \\
\hline & Female & 13.0 & $50.7 \%$ & $25.2 \%$ & $13.8 \%$ & \\
\hline \multirow[t]{2}{*}{ CA19-9 } & $<500 \mathrm{U} / \mathrm{L}$ & 22.0 & $57.5 \%$ & $30.3 \%$ & $20.9 \%$ & .092 \\
\hline & $\geq 500 \mathrm{U} / \mathrm{L}$ & 15.0 & $53.1 \%$ & $22.7 \%$ & $14.6 \%$ & \\
\hline \multirow[t]{2}{*}{ Tumor size } & $<25 \mathrm{~mm}$ & 20.0 & $63.7 \%$ & $28.7 \%$ & $20.3 \%$ & .003 \\
\hline & $\geq 25 \mathrm{~mm}$ & 10.0 & $44.9 \%$ & $21.3 \%$ & $13.2 \%$ & \\
\hline \multirow[t]{2}{*}{ Bismuth type } & I-II & 16.0 & $56.1 \%$ & $27.2 \%$ & $20.0 \%$ & .434 \\
\hline & III-IV & 17.0 & $52.5 \%$ & $22.4 \%$ & $12.9 \%$ & \\
\hline \multirow[t]{2}{*}{ Type of hepatectomy } & Minor & 18.0 & $52.6 \%$ & $23.7 \%$ & $16.2 \%$ & .808 \\
\hline & Major & 15.0 & $55.6 \%$ & $25.8 \%$ & $17.3 \%$ & \\
\hline \multirow[t]{2}{*}{ Vascular invasion } & No & 20.0 & $59.7 \%$ & $27.3 \%$ & $17.5 \%$ & .037 \\
\hline & Yes & 12.0 & $47.1 \%$ & $22.8 \%$ & $17.3 \%$ & \\
\hline \multirow[t]{2}{*}{ Perineural invasion } & No & 16.0 & $54.9 \%$ & $28.3 \%$ & $18.7 \%$ & .382 \\
\hline & Yes & 14.0 & $54.1 \%$ & $18.8 \%$ & $12.9 \%$ & \\
\hline \multirow[t]{2}{*}{ Differentiation } & Well-moderated & 18.0 & $59.4 \%$ & $27.6 \%$ & $18.1 \%$ & .003 \\
\hline & Poor & 8.0 & $31.3 \%$ & $12.3 \%$ & $12.3 \%$ & \\
\hline \multirow[t]{2}{*}{ LN status } & Negative (N0) & 20.0 & $60.4 \%$ & $30.9 \%$ & $20.6 \%$ & .001 \\
\hline & Positive $(\mathrm{N}+)$ & 10.0 & $44.9 \%$ & $14.7 \%$ & $10.7 \%$ & \\
\hline
\end{tabular}

$P, P$ value of the log-rank test

RFS, recurrence-free survival; CA19-9, carbohydrate antigen 19-9; $L N$, lymph node
$\mathrm{CI}=1.187-2.780, P=0.006)$ were independent risk factors affecting RFS (Table 5).

\section{Comparing the Predictive Ability of the LN Staging System}

Figure 2A, C, and E, respectively, report the cross-validation ROC curve analysis results of various methods of LN staging for OS 1, 3, and 5 years after surgery. In 1-year OS, the variable having the highest AUC was LODDS (AUC $=0.640$, $95 \% \mathrm{CI}=0.561-0.719, P=0.001$ ). In 3 -year OS, the variable having the highest AUC was LODDS (AUC $=0.595$, $95 \% \mathrm{CI}=0.508-0.682, P=0.045)$. In 5-year OS, the variable having the highest AUC was LODDS (AUC $=0.683,95 \%$ $\mathrm{CI}=0.571-0.794, P=0.005)$. Figure $2 \mathrm{~B}, \mathrm{D}$, and $\mathrm{F}$, respectively, report the cross-validation analysis of the ROC curve of different methods of LN staging for RFS 1, 3, and 5 years after surgery. In 1-year RFS, the variable having the highest AUC was LODDS (AUC $=0.663,95 \% \mathrm{CI}=0.539-0.734$, $P \leq 0.001)$. In 3-year RFS, the variable having the highest AUC was LODDS (AUC $=0.603,95 \% \mathrm{CI}=0.506-0.690$, $P=0.047)$. In 5-year RFS, the variable having the highest AUC was LODDS (AUC $=0.671,95 \% \mathrm{CI}=0.542-0.799$, $P=0.019)$. The AUC of LODDS, LNR, MLN, AJCC $\mathrm{pN}$ stage, and LN status at 1-year, 3-year, and 5-year time point was in Supplement Table 1.

In 1-year OS, the cut-off value of LODDS is -0.667896 . In 3-year OS, the cut-off value of LODDS was -0.520696 . In 5-year OS, the cut-off value of LODDS was -0.823365 . In 1-year RFS, the cut-off value of LODDS was -0.520696 . In 3-year RFS, the cut-off value of LODDS was -0.667896 . In 5-year RFS, the cut-off value of LODDS was -0.823365 .

\section{Discussion}

HCCA is the most common type of cholangiocarcinoma. Currently, surgical resection is the only curable method. LN metastasis has been shown to be a key factor in evaluating the prognosis of patients with cholangiocarcinoma undergoing radical surgery ${ }^{24,25}$. The AJCC LN staging, 8th edition, is defined according to the number of LN metastasis areas, without considering the LN metastases number. Many studies believe that to assess cholangiocarcinoma prognosis based on the number of LN metastasis areas is controversial 26,27 . Studies have pointed out that to evaluating the location of LN metastasis is not sufficient. The number of LN metastases also has a direct impact on the prognosis of patients ${ }^{28}$. In this study, we also got similar results. We found that 

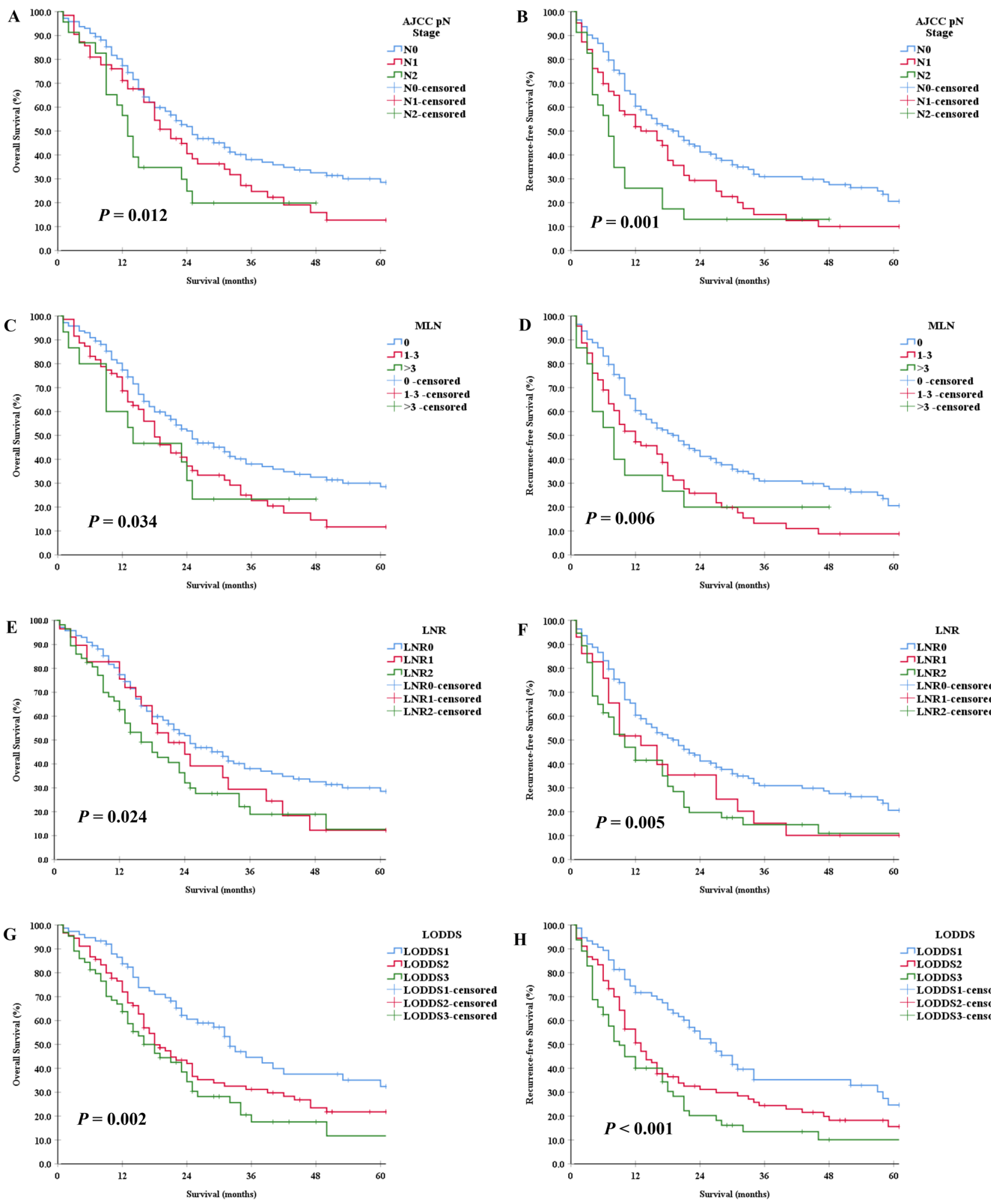

Fig. 1 Kaplan-Meier curves of OS and RFS in patients with hilar cholangiocarcinoma underwent surgery by AJCC pN stage (OS, A; RFS, B), MLN (OS, C; RFS, D), LNR (OS, E; RFS, F), and LODDS (OS, G; RFS, H). OS, overall survival; RFS, recurrence-free survival;

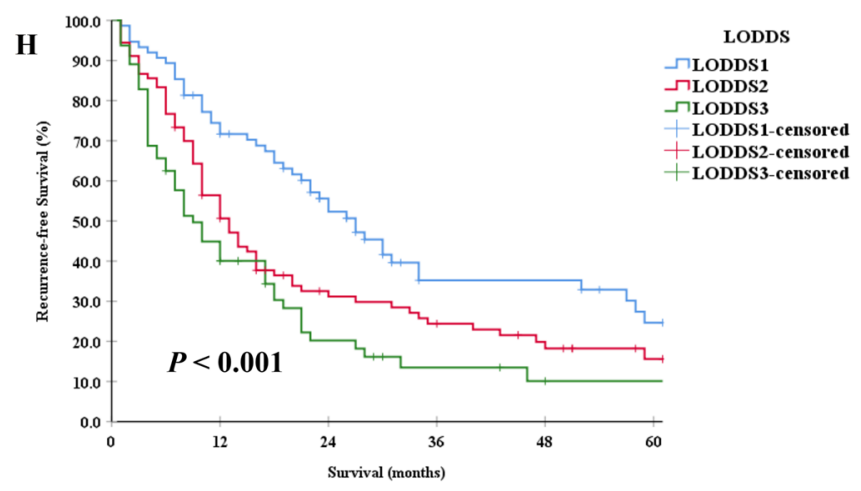

AJCC, American Joint Committee on Cancer; MLN, number of metastatic LNs; LNR, lymph node ratio; LODDS, log odds of metastatic lymph node 
Table 4 Kaplan-Meier analysis and multivariable Cox regression survival analysis for overall survival in the 229 patients with hilar cholangiocarcinoma according to each LN staging methods

\begin{tabular}{|c|c|c|c|c|c|c|c|c|}
\hline \multicolumn{2}{|c|}{ LN staging methods } & \multicolumn{5}{|c|}{ Kaplan-Meier univariate analysis } & \multicolumn{2}{|c|}{ Cox regression analysis } \\
\hline & & $\begin{array}{l}\text { Median OS } \\
\text { (months) }\end{array}$ & 1-year OS & 3-year OS & 5-year OS & $\mathrm{P}$ & $\mathrm{HR}^{*}(95 \% \mathrm{CI})$ & $P$ \\
\hline \multirow[t]{2}{*}{ LN status } & Negative (N0) & 25.0 & $77.4 \%$ & $38.1 \%$ & $28.5 \%$ & .010 & Ref & \\
\hline & Positive $(\mathrm{N}+)$ & 18.0 & $67.1 \%$ & $22.6 \%$ & $12.6 \%$ & & $1.363(1.125-1.917)$ & .041 \\
\hline \multirow[t]{3}{*}{ AJCC pN stage } & 0 & 25.0 & $77.4 \%$ & $38.1 \%$ & $28.5 \%$ & .012 & Ref & \\
\hline & 1 & 21.0 & $71.1 \%$ & $27.2 \%$ & $12.7 \%$ & & $1.271(0.877-1.843)$ & .206 \\
\hline & 2 & 13.0 & $56.5 \%$ & $19.9 \%$ & - & & $1.716(1.001-2.940)$ & .049 \\
\hline \multirow[t]{3}{*}{ MLN } & 0 & 25.0 & $77.4 \%$ & $38.1 \%$ & $28.5 \%$ & .034 & Ref & \\
\hline & $1-3$ & 18.0 & $68.6 \%$ & $22.7 \%$ & $11.7 \%$ & & $1.352(0.948-1.929)$ & .096 \\
\hline & $>3$ & 14.0 & $60.0 \%$ & $23.3 \%$ & - & & $1.430(0.741-2.760)$ & .286 \\
\hline \multirow[t]{3}{*}{ LNR } & LNR0 (0) & 25.0 & $77.4 \%$ & $38.1 \%$ & $28.5 \%$ & .024 & Ref & \\
\hline & LNR1 (0.01-0.25) & 21.0 & $75.6 \%$ & $29.4 \%$ & $12.2 \%$ & & $1.157(0.708-1.890)$ & .561 \\
\hline & LNR2 (>0.25) & 16.0 & $62.7 \%$ & $18.9 \%$ & $12.6 \%$ & & $1.514(1.018-2.250)$ & .040 \\
\hline \multirow[t]{3}{*}{ LODDS } & LODDS1 $(\leq-0.85)$ & 32.0 & $83.7 \%$ & $44.6 \%$ & $32.4 \%$ & .002 & Ref & \\
\hline & LODDS2 $(-0.84--0.45)$ & 18.0 & $72.0 \%$ & $31.2 \%$ & $21.8 \%$ & & $1.303(0.870-1.950)$ & .199 \\
\hline & LODDS3 $(>-0.45)$ & 16.0 & $63.7 \%$ & $17.6 \%$ & $11.7 \%$ & & $1.738(1.115-2.709)$ & .015 \\
\hline
\end{tabular}

* adjusted by carbohydrate antigen 19-9, tumor size, vascular invasion, radicality, and differentiation

$O S$, overall survival; $H R$, hazard ratio; $C I$, confidence interval; Ref, reference; $L N$, lymph node; AJCC, American Joint Committee on Cancer; $M L N$, number of metastatic LNs; $L N R$, lymph node ratio; $L O D D S$, log odds of metastatic lymph node

in assessing patients' prognoses, univariate results suggest that AJCC LN staging pN0, pN1, pN2 HCCA patients, their postoperative OS, and RFS were statistically different (OS,
$P=0.012 ; \mathrm{RFS}, P=0.001)$. HCCA patients with MLN 0, $1-3$, and $>3$ also had statistical differences in their postoperative OS $(P=0.034)$ and RFS $(P=0.002)$. The results of

Table 5 Kaplan-Meier analysis and multivariable Cox regression survival analysis for recurrence-free survival in the 229 patients with hilar cholangiocarcinoma according to each LN staging methods

\begin{tabular}{|c|c|c|c|c|c|c|c|c|}
\hline \multicolumn{2}{|c|}{ LN staging methods } & \multicolumn{5}{|c|}{ Kaplan-Meier univariate analysis } & \multicolumn{2}{|c|}{ Cox regression analysis } \\
\hline & & $\begin{array}{l}\text { Median RFS } \\
\text { (months) }\end{array}$ & 1-year RFS & 3-year RFS & 5-year RFS & $P$ & $\mathrm{HR}^{*}(95 \% \mathrm{CI})$ & $P$ \\
\hline \multirow[t]{2}{*}{ LN status } & Negative (N0) & 20.0 & $60.4 \%$ & $30.9 \%$ & $20.6 \%$ & .001 & Ref & \\
\hline & Positive $(\mathrm{N}+)$ & 10.0 & $44.9 \%$ & $14.7 \%$ & $10.7 \%$ & & $1.486(1.073-2.059)$ & .017 \\
\hline \multirow[t]{3}{*}{ AJCC pN stage } & 0 & 20.0 & $60.4 \%$ & $30.9 \%$ & $20.6 \%$ & .001 & Ref & \\
\hline & 1 & 13.0 & $51.8 \%$ & $15.0 \%$ & $10.0 \%$ & & $1.378(0.966-1.968)$ & .077 \\
\hline & 2 & 7.0 & $26.1 \%$ & $13.0 \%$ & - & & $1.891(1.138-3.143)$ & .014 \\
\hline \multirow[t]{3}{*}{ MLN } & 0 & 20.0 & $60.4 \%$ & $30.9 \%$ & $20.6 \%$ & .006 & Ref & \\
\hline & $1-3$ & 12.0 & $47.3 \%$ & $13.2 \%$ & $8.8 \%$ & & $1.478(1.052-2.078)$ & .024 \\
\hline & $>3$ & 8.0 & $33.3 \%$ & $20.0 \%$ & - & & $1.532(0.820-2.863)$ & .181 \\
\hline \multirow[t]{3}{*}{ LNR } & LNR0 (0) & 20.0 & $60.4 \%$ & $30.9 \%$ & $20.6 \%$ & .005 & Ref & \\
\hline & LNR1 (0.01-0.25) & 13.0 & $51.7 \%$ & $15.2 \%$ & $10.1 \%$ & & $1.455(0.913-2.319)$ & .115 \\
\hline & LNR2 $(>0.25)$ & 10.0 & $41.5 \%$ & $14.6 \%$ & $10.9 \%$ & & $1.505(1.030-2.200)$ & .035 \\
\hline \multirow[t]{3}{*}{ LODDS } & LODDS $1(\leq-0.85)$ & 27.0 & $71.7 \%$ & $35.2 \%$ & $24.7 \%$ & $<.001$ & Ref & \\
\hline & LODDS2 $(-0.84--0.45)$ & 13.0 & $50.7 \%$ & $24.4 \%$ & $15.6 \%$ & & $1.360(0.927-1.996)$ & .116 \\
\hline & LODDS3 $(>-0.45)$ & 9.0 & $40.1 \%$ & $13.5 \%$ & $10.1 \%$ & & $1.817(1.187-2.780)$ & .006 \\
\hline
\end{tabular}

* adjusted by carbohydrate antigen 19-9, tumor size, vascular invasion, radicality, and differentiation

$R F S$, recurrence-free survival; $H R$, hazard ratio; $C I$, confidence interval; Ref, reference; $L N$, lymph node; AJCC, American Joint Committee on Cancer; $M L N$, number of metastatic LNs; $L N R$, lymph node ratio; $L O D D S$, log odds of metastatic lymph node 
1-year OS

A

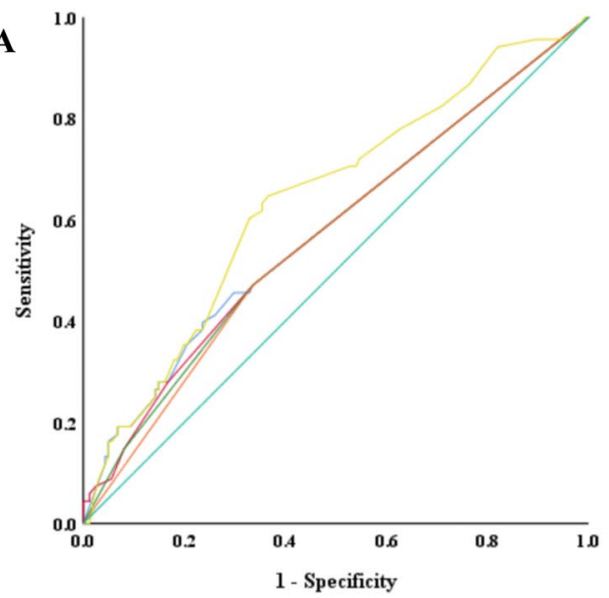

3-year OS

C

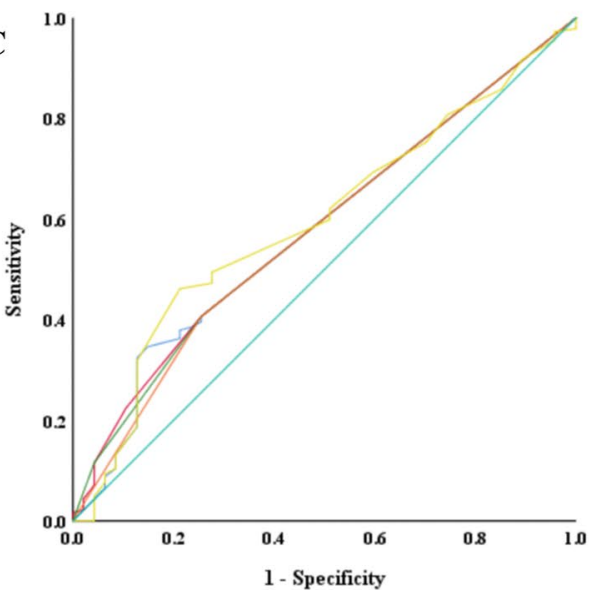

1-year RFS

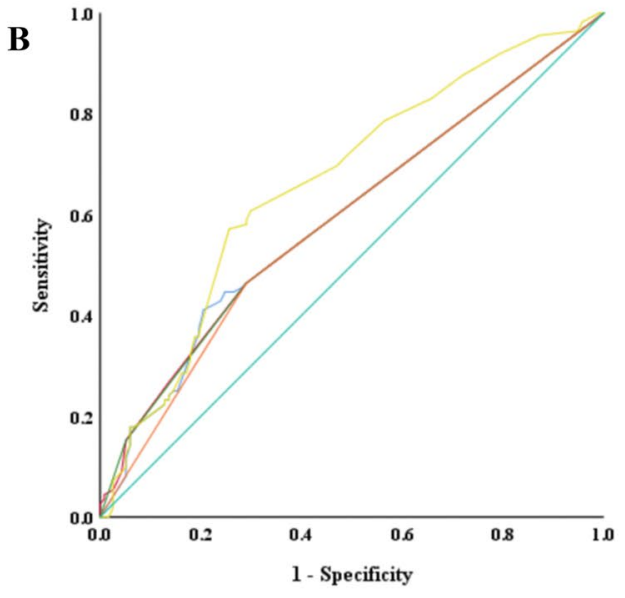

3-year RFS
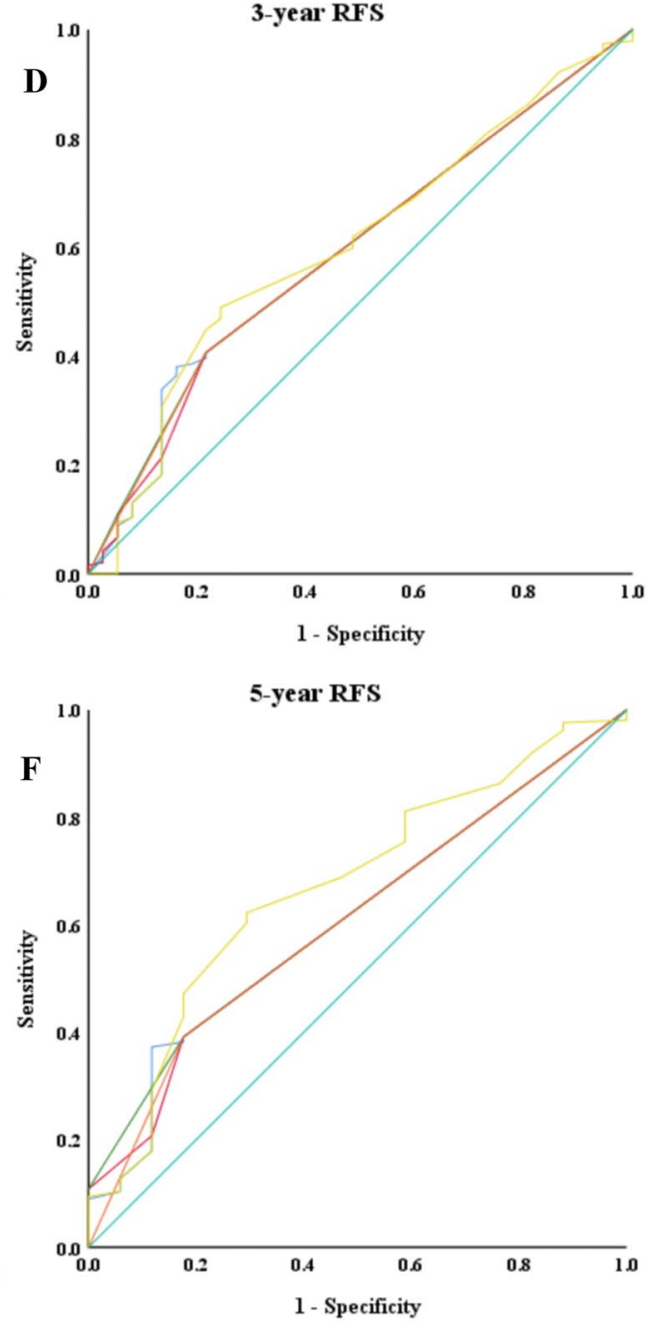

LNR

$-\mathrm{MLN}$

AJCC pN Stage

LN status

LODDS

Reference Line 5-year OS

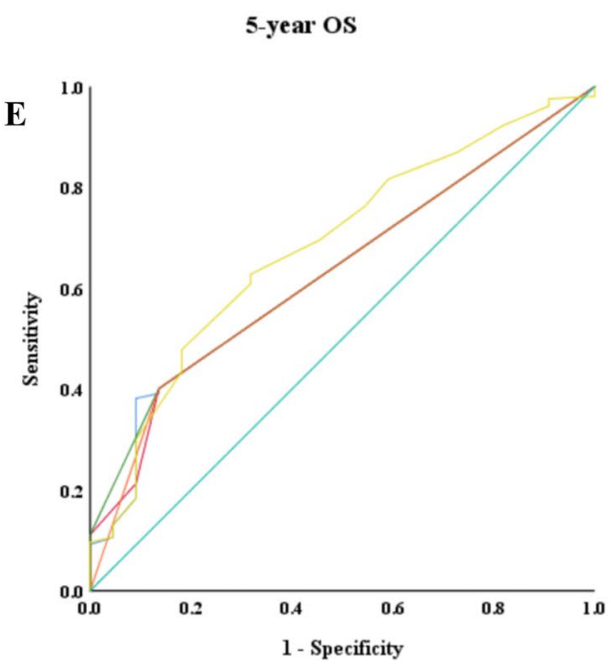

Fig. 2 Comparison for the AUC of LODDS, LNR, MLN, AJCC pN stage, and LN status according to 1-year OS (A), 3-year OS (C), 5-year OS (E), 1-year RFS (B), 3-year RFS (D), and 5-year RFS (F). AUC, area under the curve; OS, overall survival; RFS, recurrence- free survival; LN, lymph node; AJCC, American Joint Committee on Cancer; MLN, number of metastatic LNs; LNR, lymph node ratio; LODDS, log odds of metastatic lymph node 
this study believe that the location and number of LN metastases can affect patients' prognosis. To further explore the effect of the number of LN metastases on the prognosis, we tried to use more methods according to the LN metastases number to evaluate the patient's OS and RFS, such as LNR and LODDS, to estimate the best LN staging system for evaluating the patient's prognosis.

Many scholars believe that the sufficient LN examination number is the prerequisite for the accurate number of positive LNs in gastrointestinal tumors ${ }^{29-33}$. A large-scale study collected the clinicopathological data of 20,068 patients with gallbladder cancer, ampullary cancer, and extrahepatic bile ducts from the SEER Cancer Registry. More than $10 \mathrm{LN}$ examinations are needed to accurately stage the LNs ${ }^{34}$. However, it is controversial that expanding the scope of lymph node dissection is beneficial to patients with HCCA. Hakeem et al. indicated that the prognosis of HCCA patients with more than 20 lymphatic examinations was worse ${ }^{6}$. They believed that more lymph node examinations may not improve the prognosis of patients with hilar cholangiocarcinoma after surgery. The more LNs checked, the more traumatic the operation was. Our team believes that the most suitable system for LN staging should be found in the limited number of LN examinations. Moreover, it should be accurate to assess the prognosis of patients and avoid deliberately taking more LNs for examination. The median of the number of LN examinations in this study was only 3 (range, 1-9).

The number of LNs that were positive increases as the number of $\mathrm{LN}$ examinations increases. Therefore, it is inaccurate to estimate the prognosis based alone on the number of positive LNs. LODDS and LNR are the systems of LN staging according to the number of positive LNs proposed in recent years. As per various studies, LODDS and LNR are more reliable in assessing the prognosis of gastrointestinal tumors $11,13,15,16,35-37$.

In the current study, the number of positive LNs, AJCC LN staging, LODDS, and LNR were used to assess the OS of HCCA patients who underwent radical surgery. Our results indicate that the AUC of LODDS was significantly higher than other LN staging systems when evaluating patients with 1-, 3-, and 5-year OS. This also suggests that LODDS was found to be the best $\mathrm{LN}$ staging system for predicting patient mortality.

At present, studies have pointed out that LODDS has better predictive performance in the assessment of gastrointestinal cancer. Zhou et al. analyzed the clinicopathological data of 1999 patients retrospectively with distant metastatic gastric cancer and pointed out that when predicting 1-year cancer-specific survival (CSS) and 2-year CSS, compared with MLN and LNR, LODDS had the highest AUC ${ }^{18}$. In addition, there are also studies comparing different LN analysis systems in evaluating the prognosis of HCCA patients undergoing radical surgery. Conci et al. compared the predictive capacity of LODDS, MLN, and LNR on OS in 99 HCCA patients. They believe that LODDS has the highest AUC when predicting 3-year OS ${ }^{19}$. Therefore, Conci believes that LODDS is the best LN staging system when predicting patient mortality. After analyzing the clinicopathological data of 437 HCCA patients, Bagante et al. concluded that a minimum of four LNs should be assessed for HCCA patients undergoing radical surgery ${ }^{38}$. When the number of ELNs is greater than 4, LODDS shows better prediction performance than the AJCC $\mathrm{pN}$ stage for evaluating $\mathrm{OS}^{38}$. However, none of the above reports on the prognostic relationship between LODDS and HCCA mentioned the relationship between the system of $\mathrm{LN}$ staging and tumor recurrence. We hypothesize that if a different $\mathrm{LN}$ staging system is used to evaluate the tumor recurrence of HCCA patients, that is, to evaluate the patient's RFS, whether the same conclusions as the evaluation of OS can be obtained, that is, LODDS is the best LN staging system for preciting the mortality rate of HCCA. Based on this, we used LODDS, LNR, the number of positive LNs, and AJCC LN staging to evaluate the 1-, 3-, and 5-years RFS of HCCA patients and found that the AUC of LODDS was the highest. Therefore, we conclude that LODDS is the best LN staging system for predicting the recurrence rate of HCCA patients.

This study has the following limitations. Due to the retrospective nature, all collected data were biased. This study was like other earlier reported studies conducted at single centers. In addition, the pathological results of each case in this study were examined by the different hepatobiliary pathology team while using the same pathological examination process. At the same time, our study lacked external verification. Some variables that are considered critical to predicting prognosis were missing, such as comorbidity, microvascular invasion, or patients' performance status. However, the results of the current study can improve the debatable issue of the LN staging system in HCCA.

\section{Conclusions}

In summary, LN metastasis is a key factor in judging prognosis. LODDS, on the basis of number of positive LNs, seems to be the best $\mathrm{LN}$ staging system for predicting the mortality and recurrence rate of HCCA patients after radical resection. In addition, proper $\mathrm{LN}$ dissection is of great significance for clarifying tumor staging and selecting treatment methods.

Abbreviations IQR: Interquartile range; OS: Overall survival; RFS: Recurrence-free survival; AUC: Area under the curve; HR: Hazard ratio; CI: Confidence interval; Ref: Reference; LN: Lymph node; 
CA19-9: Carbohydrate antigen 19-9; AJCC: American Joint Committee on Cancer; MLN: Number of metastatic LNs; LNR: Lymph node ratio; LODDS: Log odds of metastatic lymph node

Supplementary Information The online version contains supplementary material available at https://doi.org/10.1007/s11605-021-05211-x.

Author Contribution ZP Liu designed and performed the research and wrote the paper; ZY Chen and HS Dai designed the research and supervised the report; QY Zhang and WY Chen designed the research and contributed to the analysis; HS Dai, YY Huang, QY Zhang, Y Gong, Y Jiang, and J Bai provided clinical advice; HS Dai, ZP Liu, and QY Zhang supervised the report.

Funding This study was supported by the National Natural Science Foundation of China, 81874211, and personalized training of key support objects for the talent people of the Army Medical University, XZ-2019-505-014.

\section{Declarations}

Conflict of Interest The authors declare no competing interests.

Open Access This article is licensed under a Creative Commons Attribution 4.0 International License, which permits use, sharing, adaptation, distribution and reproduction in any medium or format, as long as you give appropriate credit to the original author(s) and the source, provide a link to the Creative Commons licence, and indicate if changes were made. The images or other third party material in this article are included in the article's Creative Commons licence, unless indicated otherwise in a credit line to the material. If material is not included in the article's Creative Commons licence and your intended use is not permitted by statutory regulation or exceeds the permitted use, you will need to obtain permission directly from the copyright holder. To view a copy of this licence, visit http://creativecommons.org/licenses/by/4.0/.

\section{References}

1. Matull WR, Khan SA, Pereira SP. Re: Impact of classification of hilar cholangiocarcinomas (Klatskin tumors) on incidence of intra- and extrahepatic cholangiocarcinoma in the United States. J Natl Cancer Inst 2007;99 (5):407; author reply 407-8

2. 2.Zhou $\mathrm{R}, \mathrm{Wu} \mathrm{Z}$, Zhang J, et al. Clinical significance of accurate identification of lymph node status in distant metastatic gastric cancer. Oncotarget 2016;7(1):1029-41.

3. Ke B, Song XN, Liu N, Zhang RP, Wang CL, Liang H. Prognostic value of the lymph node ratio in stage III gastric cancer patients undergoing radical resection. PLoS One 2014;9(5):e96455.

4. Kow AW, Wook CD, Song SC, et al. Role of caudate lobectomy in type III A and III B hilar cholangiocarcinoma: a 15-year experience in a tertiary institution. World J Surg 2012;36(5):1112-21.

5. de Jong MC, Marques H, Clary BM, et al. The impact of portal vein resection on outcomes for hilar cholangiocarcinoma: a multiinstitutional analysis of 305 cases. Cancer 2012;118(19):4737-47.

6. Hakeem AR, Marangoni G, Chapman SJ, et al. Does the extent of lymphadenectomy, number of lymph nodes, positive lymph node ratio and neutrophil-lymphocyte ratio impact surgical outcome of perihilar cholangiocarcinoma. Eur J Gastroenterol Hepatol 2014;26(9):1047-54.

7. Lin Z, Chen W, Chen Y, et al. A new classification of lymph node metastases according to the lymph node stations for predicting prognosis in surgical patients with esophageal squamous cell carcinoma. Oncotarget 2016;7(46):76261-73.

8. Guglielmi A, Ruzzenente A, Campagnaro T, et al. Patterns and prognostic significance of lymph node dissection for surgical treatment of perihilar and intrahepatic cholangiocarcinoma. J Gastrointest Surg 2013;17(11):1917-28.

9. Gaspersz MP, Buettner S, van Vugt J, et al. Evaluation of the New American Joint Committee on Cancer Staging Manual 8th Edition for Perihilar Cholangiocarcinoma. J Gastrointest Surg 2020;24(7):1612-8.

10. Lee JW, Lee JH, Park Y, et al. Prognostic Predictability of American Joint Committee on Cancer 8th Staging System for Perihilar Cholangiocarcinoma: Limited Improvement Compared with the 7th Staging System. Cancer Res Treat 2020;52(3):886-95.

11. Guglielmi A, Ruzzenente A, Campagnaro T, et al. Prognostic significance of lymph node ratio after resection of peri-hilar cholangiocarcinoma. HPB (Oxford) 2011;13(4):240-5.

12. Sakata J, Wakai T, Matsuda Y, et al. Comparison of Number Versus Ratio of Positive Lymph Nodes in the Assessment of Lymph Node Status in Extrahepatic Cholangiocarcinoma. Ann Surg Oncol 2016;23(1):225-34.

13. Tamandl D, Kaczirek K, Gruenberger B, et al. Lymph node ratio after curative surgery for intrahepatic cholangiocarcinoma. Br J Surg 2009;96(8):919-25.

14. Spolverato G, Ejaz A, Kim Y, et al. Prognostic Performance of Different Lymph Node Staging Systems After Curative Intent Resection for Gastric Adenocarcinoma. Ann Surg 2015;262(6):991-8.

15. Kim Y, Spolverato G, Amini N, et al. Surgical Management of Intrahepatic Cholangiocarcinoma: Defining an Optimal Prognostic Lymph Node Stratification Schema. Ann Surg Oncol 2015;22(8):2772-8.

16. Aurello P, Petrucciani N, Nigri GR, et al. Log odds of positive lymph nodes (LODDS): what are their role in the prognostic assessment of gastric adenocarcinoma. J Gastrointest Surg 2014;18(7):1254-60.

17. Kwon J, Kim K, Chie EK, et al. Prognostic relevance of lymph node status for patients with ampullary adenocarcinoma after radical resection followed by adjuvant treatment. Eur J Surg Oncol 2017;43(9):1690-6.

18. Zhou R, Zhang J, Sun H, Liao Y, Liao W. Comparison of three lymph node classifications for survival prediction in distant metastatic gastric cancer. Int J Surg 2016;35:165-71.

19. Conci S, Ruzzenente A, Sandri M, et al. What is the most accurate lymph node staging method for perihilar cholangiocarcinoma? Comparison of UICC/AJCC $\mathrm{pN}$ stage, number of metastatic lymph nodes, lymph node ratio, and log odds of metastatic lymph nodes. Eur J Surg Oncol 2017;43(4):743-50.

20. Bismuth H, Corlette MB. Intrahepatic cholangioenteric anastomosis in carcinoma of the hilus of the liver. Surg Gynecol Obstet 1975;140(2):170-8.

21. Miyazaki M, Ohtsuka M, Miyakawa S, et al. Classification of biliary tract cancers established by the Japanese Society of HepatoBiliary-Pancreatic Surgery: 3(rd) English edition. J Hepatobiliary Pancreat Sci 2015;22(3):181-96.

22. Wu SG, Sun JY, Yang LC, et al. Prognosis of patients with esophageal squamous cell carcinoma after esophagectomy using the log odds of positive lymph nodes. Oncotarget 2015;6(34):36911-22.

23. Wang JK, Hu HJ, Shrestha A, et al. Can preoperative and postoperative CA19-9 levels predict survival and early recurrence in patients with resectable hilar cholangiocarcinoma. Oncotarget 2017;8(28):45335-44.

24. Ito F, Agni R, Rettammel RJ, et al. Resection of hilar cholangiocarcinoma: concomitant liver resection decreases hepatic recurrence. Ann Surg 2008;248(2):273-9.

25. Furusawa N, Kobayashi A, Yokoyama T, Shimizu A, Motoyama H, Miyagawa S. Surgical treatment of 144 cases of hilar 
cholangiocarcinoma without liver-related mortality. World J Surg 2014;38(5):1164-76.

26. Bertero L, Massa F, Metovic J, et al. Eighth Edition of the UICC Classification of Malignant Tumours: an overview of the changes in the pathological TNM classification criteria-What has changed and why. Virchows Arch 2018;472(4):519-31.

27. Teramoto N, Tanimizu M, Nishimura R. Present situation of pTNM classification in Japan: questionnaire survey of the pathologists of Gan-shinryo-renkei-kyoten Byoin (local core cancer hospitals) on pTNM classification. Pathol Int 2009;59(3):167-74.

28. Mao K, Liu J, Sun J, et al. Patterns and prognostic value of lymph node dissection for resected perihilar cholangiocarcinoma. J Gastroenterol Hepatol 2016;31(2):417-26.

29. Sekiya S, Suzuki A. Intrahepatic cholangiocarcinoma can arise from Notch-mediated conversion of hepatocytes. J Clin Invest 2012;122(11):3914-8.

30. Bergquist A, von Seth E. Epidemiology of cholangiocarcinoma. Best Pract Res Clin Gastroenterol 2015;29(2):221-32.

31. Valero V 3rd, Cosgrove D, Herman JM, Pawlik TM. Management of perihilar cholangiocarcinoma in the era of multimodal therapy. Expert Rev Gastroenterol Hepatol 2012;6(4):481-95.

32. DeOliveira ML, Cunningham SC, Cameron JL, et al. Cholangiocarcinoma: thirty-one-year experience with 564 patients at a single institution. Ann Surg 2007;245(5):755-62.

33. Siegel RL, Miller KD, Jemal A. Cancer statistics, 2019. CA Cancer J Clin 2019;69(1):7-34.
34. Schwarz RE, Smith DD. Lymph node dissection impact on staging and survival of extrahepatic cholangiocarcinomas, based on U.S. population data. J Gastrointest Surg 2007;11(2):158-65.

35. Marchet A, Mocellin S, Ambrosi A, et al. The ratio between metastatic and examined lymph nodes ( $\mathrm{N}$ ratio) is an independent prognostic factor in gastric cancer regardless of the type of lymphadenectomy: results from an Italian multicentric study in 1853 patients. Ann Surg 2007;245(4):543-52.

36. Wang X, Appleby DH, Zhang X, Gan L, Wang JJ, Wan F. Comparison of three lymph node staging schemes for predicting outcome in patients with gastric cancer. Br J Surg 2013;100(4):505-14.

37. Gleisner AL, Mogal H, Dodson R, et al. Nodal status, number of lymph nodes examined, and lymph node ratio: what defines prognosis after resection of colon adenocarcinoma. J Am Coll Surg 2013;217(6):1090-100.

38. Bagante F, Tran T, Spolverato G, et al. Perihilar Cholangiocarcinoma: Number of Nodes Examined and Optimal Lymph Node Prognostic Scheme. J Am Coll Surg 2016;222(5):750-9.e2.

Publisher's Note Springer Nature remains neutral with regard to jurisdictional claims in published maps and institutional affiliations. 